\title{
Zeta-factor Determination Using Metal Thin Films for STEM-SDD Compositional Analysis of Iron-based Al- loy Systems
}

\author{
Keiko Yamada, ${ }^{\mathrm{a}, \mathrm{b}, \dagger}$ Yuya Murata, ${ }^{\mathrm{a}}$ Taku Moronaga, ${ }^{\mathrm{c}}$ Kazushi Hayashi, ${ }^{\mathrm{a}}$ Chikara Ichihara, ${ }^{\mathrm{a}}$ Toru Hara ${ }^{\mathrm{b}, \mathrm{c}}$ \\ a Applied Physics Research Laboratory, Kobe Steel, Ltd., 1-5-5, Takatsukadai, Nishi-ku, Kobe, 651-2271, Japan \\ ${ }^{\mathrm{b}}$ Department of Applied Science for Electronics and Materials, Kyushu University, 6-1, Kasugakoen, Kasuga, 816-8580, Japan \\ ${ }^{c}$ Research Network and Facility Services Division, National Institute for Materials Science, 1-2-1, Sengen, Tsukuba, 305-0047, Japan \\ $\dagger$ Corresponding author: yamada.keiko@kobelco.com
}

Received: 10 December, 2019, Accepted 12 February, 2020, Published 19 March, 2020

The zeta $(\zeta)$ factor proposed by Watanabe and Williams [M. Watanabe and D. B. Williams, J. Microsc. 221, 89 (2006)] is a fundamental parameter for quantitative X-ray analysis with consideration of X-ray absorption using scanning transmission electron microscopy in combination with silicon drift detectors (STEM-SDD). In this study, the $\zeta$-factors for Fe and $\mathrm{Mn}$ in ironbased alloy systems were determined by two thin-film approaches, namely, the "lift-off method" and the "direct deposition method". The Mn content in the Fe-Mn alloy obtained by using the $\zeta$-factor, which was measured with Mn thin films directly deposited on a transmission electron microscopy (TEM) mesh grid, was in good agreement with the composition determined by fluorescent X-ray analysis. Determination of the accurate film thicknesses is essential for the accurate measurement of the $\zeta$-factors. A layered structure and a directly deposited thin film is found effective for a standard sample to prevent bending and overlapping of samples. It is proved that the proposed method to fabricate the standard sample is useful for quantitative X-ray analysis with existing STEMSDD system.

Keywords STEM-SDD; Quantitative X-ray analysis; $\zeta$-factor method; Cliff-Lorimer ratio method

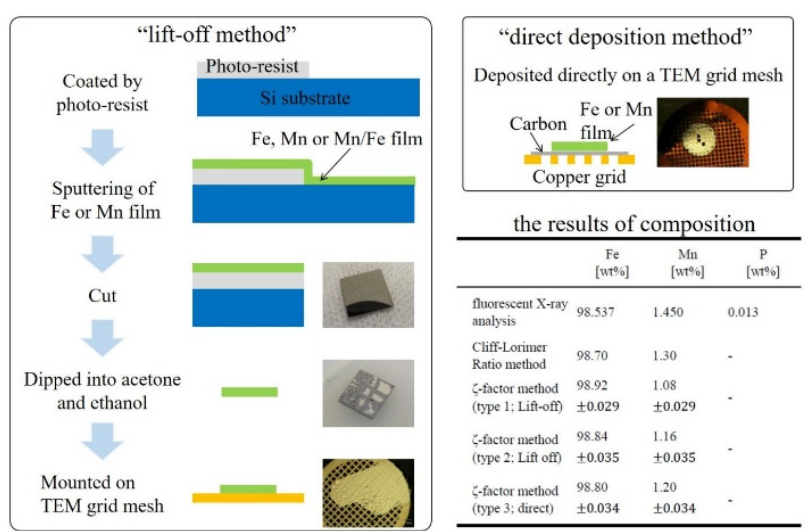

\section{INTRODUCTION}

In iron and steels, which are one of the most common materials in many industries, controlling contents of additive elements is crucial since they change their mechanical properties. Among them, phosphorus and sulfur are typical light elements which make steels brittle [1-5]. Such light elements tend to segregate and/or precipitate at grain boundaries or on surfaces. Therefore, analysis with a scanning transmission electron microscope (STEM) and a conventional energy-dispersive X-ray spectrometer (EDX) is favorably applied to measure contents of additive elements in steels. 
Watanabe and Williams [7].

The $\zeta$-factor is defined by the following equation as,

$$
\rho t=\zeta_{\mathrm{A}} \frac{I_{\mathrm{A}} A_{\mathrm{A}}}{C_{\mathrm{A}} D_{\mathrm{e}}}
$$

where $\rho$ is the density, $t$ the thickness, $I_{\mathrm{A}}$ the X-ray intensity (net counts), $C_{\mathrm{A}}$ the concentration, $D_{\mathrm{e}}$ the total electron dose, and $A_{\mathrm{A}}$ the correction factor for X-ray absorption of element A. The $\zeta$-factor for each element can be calculated by these five parameters in Eq. (1). Once the $\zeta$-factors are determined, the chemical composition of each element is given with consideration of X-ray absorption by the following:

$$
\begin{gathered}
C_{j}=\frac{\zeta_{j} I_{j} A_{j}}{\sum_{i} \zeta_{i} I_{i} A_{i}} \\
\sum_{i} C_{i}=1
\end{gathered}
$$

It is obvious that the $\zeta$-factor method is effective only when an appropriated standard sample is available.

Previous studies have proposed fabrication methods of standard samples to determine $\zeta$-factors. For instance, Watanabe and Willams used the National Institute of Standards and Technology (NIST) standard reference materials, SRM 2063 and 2063a, in which the composition, the thickness, and the density were accurately measured [7]. However, this standard sample is not available on the market now. Zanaga et al. showed that the $\zeta$-factors can be measured by pure element nanoparticles [8], but this measurement needs to utilize combination of electron tomography and EDX. Kothleitner et al. showed that the thickness of the thinfilm sample can be measured by electron energy loss spectroscopy [9], that requires a time-consuming calibration of the inelastic mean path and a needle shaped sample with the same phase of the thin film. The purpose of this study is to find a fabrication method of the standard samples in a more simple way.

In this study, two kinds of thin-film approaches were used to estimate the $\zeta$-factors of $\mathrm{Fe}$ and $\mathrm{Mn}$. The proposed approaches are application of microfabrication established in the field of microelectronics. The referenced thin films were prepared by conventional sputtering and precisely characterized by various analytical tools. To verify the measured $\zeta$-factors, we compared the chemical composition of the FeMn alloy with that obtained by fluorescent X-ray analysis. We discussed critical issues for determination of the parameters to calculate the $\zeta$-factor based on the results obtained by various techniques.

\section{MATERIALS AND METHODS}

\section{A. Sample preparation}

We estimated the $\zeta$-factors of Fe and $\mathrm{Mn}$ from thin films. Manganese is a common additive element in various steels. The metal thin films were deposited by conventional sputtering with pure $\mathrm{Fe}$ and $\mathrm{Mn}$ targets. The standard samples were (a)
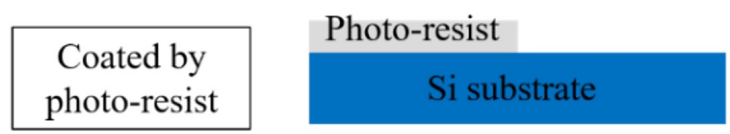

(b)

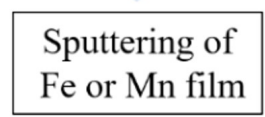

(c)

(d)
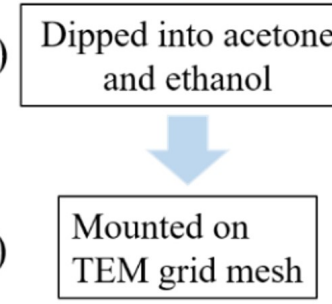

Fe or Mn film
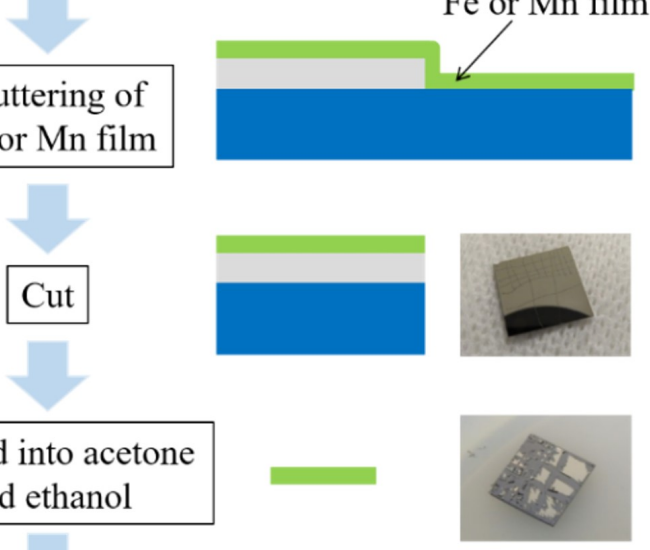

Figure 1: Preparation of a Fe or Mn thin film. (a) A Si wafer was partially covered by the photoresist. (b) Either Fe or Mn film was deposited. (c) The sample was cut into small pieces. The image is for metal thin film/photoresist/Si. (d) The sample was dipped into acetone to remove a metal film from Si by dissolving the photoresist layer (optical image) followed by ethanol for cleaning. (e) The metal film was received by a TEM grid mesh.

fabricated by the following two individual approaches.

The first approach is "lift-off method". Figure 1 shows the fabrication steps, the sectional views, and the optical images of metal thin-film specimens for standard samples. First, a half of Si wafer was covered by photoresist [Figure 1(a)]. Then, a layer of Fe or Mn was deposited simultaneously on the $\mathrm{Si}$ or the resist/Si substrate [Figure 1(b)] by DC magnetron sputtering using pure Fe or Mn target. The specimen was diced in two pieces [Figure 1(c)]. The half of the specimen consisting of metal/resist/Si was dipped into acetone to dissolve the photoresist layer to detached the films from the substrate [Figure 1(d)]. Then, the films were picked up, and dipped into ethanol for cleaning. Finally, the films were mounted on a TEM grid mesh [Figure 1(e)]. The specimens

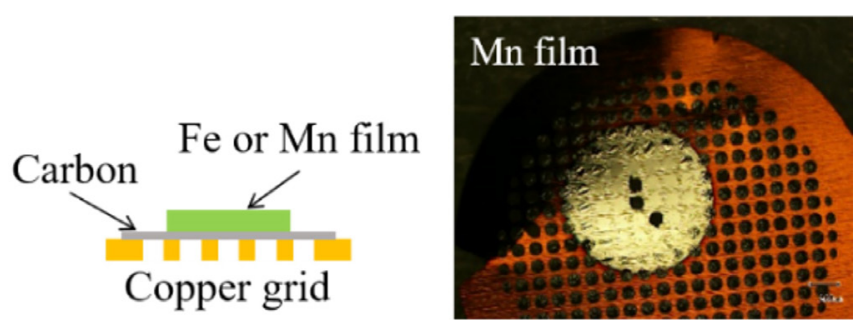

Figure 2: Sectional view of the Fe or Mn thin film which was deposited on a TEM grid mesh with a carbon-supporting film (left). Optical image of the Mn film on the grid (right). 
Table 1: Density of the Fe film obtained by XRR.

\begin{tabular}{lcccc}
\hline & $\begin{array}{c}\text { Density } \\
\left(\mathrm{g} \mathrm{cm}^{-3}\right)\end{array}$ & $\begin{array}{c}\text { Thickness } \\
(\mathrm{nm})\end{array}$ & $\begin{array}{c}\text { Roughness } \\
(\mathrm{nm})\end{array}$ & $R$ \\
\hline Surface & 5.00 & 2.56 & 0.46 & 0.0119 \\
Fe layer & 8.01 & 109.37 & 0.75 & \\
$\mathrm{Si}$ & $(2.33)$ & - & 0.80 & \\
\hline
\end{tabular}

consisting of metal/Si [Figure 1(b)] were used to measure physical parameters such as their density, thickness, and concentration. The sample consisting of the $\mathrm{Mn} / \mathrm{Fe}$ bilayer was also fabricated by the consecutive sputtering of $\mathrm{Fe}$ and $\mathrm{Mn}$.

The second approach is "direct deposition method". Figure 2 shows the schematic sectional view of a Fe or Mn thin film and the optical image of the metal thin-film specimen for standard samples $(\mathrm{Mn})$. The thin films were deposited directly on a TEM grid mesh with carbon-supporting film (EM Japan, Cat No. U1015).

\section{B. Sample Characterization}

To calculate the $\zeta$-factors, the density, thickness, and concentration of each thin film was characterized by the Rutherford Backscattering Spectrometry (RBS), X-ray reflectivity (XRR), sectional images of the scanning electron microscope (SEM), and a surface profiler. The XRR and the RBS measurements have been successfully applied to study the thinfilm structures and characterize their film thickness, composition, roughness and density [10].

The RBS measurements were carried out with $2300 \mathrm{keV}$ $\mathrm{He}^{+}$ions using a HRBS500 system (Kobe Steel, Ltd.). The XRR measurements were performed using a SmartLabRapid 2 system (Rigaku) with a $\mathrm{Cu} \mathrm{K} \alpha$ radiation. The focused ion beam (FIB)-SEM (Thermo Fisher Scientific) was used to measure the thickness of the thin films. A layer of platinum (Pt) was deposited onto the films to protect the surface, then cut them by the FIB with an accelerating energy of $30 \mathrm{kV}$ and measured the thickness from the sideview of SEM images. These analytical tools were applied to $\mathrm{Fe}$ and $\mathrm{Mn}$ single thin films made by "lift-off method". For $\mathrm{Mn} / \mathrm{Fe}$ belayed thin films ("lift-off method"), the thickness was determined by the deposition rate of each thin film. For the Fe and Mn films fabricated by the "direct deposition method", a surface profiler (KLA-Tencor) was utilized to measure the thickness.

The X-ray intensity and the total electron dose were examined by STEM-EDX and a Faraday cup installed in STEM. The STEM-EDX measurements were performed using a JEOL JEM 2800 operated at $200 \mathrm{kV}$ with a silicon drift detector (SDD). The total electron dose measured by Faraday cup was $0.97 \mathrm{nA}$. For each measured spot, EDX spectra were detected with an acquisition time of $60 \mathrm{~s}$. A sample tilt angle of $20^{\circ}$ toward the detector was chosen to minimize shadowing effects and increase the collected signal.

The net area intensity of element A $\left(I_{\mathrm{A}}\right)$ was estimated by subtracting background intensities from total area intensities. The intensities of $\mathrm{Fe}$ and $\mathrm{Mn}$ were taken from the energy
Table 2: Density of the Mn film obtained by XRR.

\begin{tabular}{lcccc}
\hline & $\begin{array}{c}\text { Density } \\
\left(\mathrm{g} \mathrm{cm}^{-3}\right)\end{array}$ & $\begin{array}{c}\text { Thickness } \\
(\mathrm{nm})\end{array}$ & $\begin{array}{c}\text { Roughness } \\
(\mathrm{nm})\end{array}$ & $R$ \\
\hline Surface & 5.59 & 6.71 & 2.04 & 0.0190 \\
Mn layer & 7.20 & 116.91 & 1.50 & \\
$\mathrm{Si}$ & $(2.33)$ & - & 0.57 & \\
\hline
\end{tabular}

ranges between $6.24 \mathrm{keV}$ and $6.56 \mathrm{keV}$ and between $5.74 \mathrm{keV}$ and $6.05 \mathrm{keV}$, respectively. The background counts are determined by averaging two intensities at lower (call B1) and higher (call B2) energy regions of the peak [11]. We used 5.68-6.10 keV (B1) and 6.60-6.92 keV (B2) for Fe and 5.35-5.66 keV (B1) and 6.07-6.38 keV (B2) for Mn. For the $\mathrm{Mn} / \mathrm{Fe}$ sample, the above calculation was not applicable because there is the peak of $\mathrm{Fe} \mathrm{K \alpha}$ in the region of B2 for Mn. Therefore, only B1 was used for the $\mathrm{Mn} / \mathrm{Fe}$ sample and $\mathrm{Fe}$ $\mathrm{Mn}$ alloy shown in Sec. III.C. The error of intensities is defined as $\Delta I_{\mathrm{A}}=\sqrt{I_{\mathrm{A}}}$.

The obtained $\zeta$-factors were tested by applying them to estimate a composition of a Fe-Mn-P alloy. The composition determined by fluorescent X-ray analysis was $98.537 \mathrm{wt} \% \mathrm{Fe}$, $1.450 \mathrm{wt} \% \mathrm{Mn}$, and $0.013 \mathrm{wt} \%$ P. Since the composition of P was well below the detection limit of the STEM-SDD system, it was not considered in the further calculation.

\section{RESULTS AND DISCUSSION}

\section{A. Measurement of physical parameters}

The density, thickness, and roughness of Fe and $\mathrm{Mn}$ on the Si substrates were examined by the XRR. Tables 1 and 2 summarize the data. In the tables, $R$ is the reliability factor defined by the following equation:

$$
R=\sqrt{\frac{\sum\left(\log y_{\text {cal }}-\log y_{\mathrm{obs}}\right)^{2}}{\left(\sum \log y_{\mathrm{obs}}\right)^{2}}},
$$

where $y_{\text {cal }}$ is the calculated value and $y_{\text {obs }}$ is the measured value. It is shown that the densities of the Fe and the Mn layers are nearly the same as those in literature as $7.87 \mathrm{~g} \mathrm{~cm}^{-3}$ and $7.21 \mathrm{~g} \mathrm{~cm}^{-3}$, respectively. The error of density $(\Delta \rho)$ measured by XRR is estimated to be $0.02 \mathrm{~g} \mathrm{~cm}^{-3}$. It means that the present thin films were close to bulk samples in terms of crystallinity. However, the roughness of the Mn film was twice bigger than that of the Fe film, and there was an unknown surface layer on both metal films. As is evident in the RBS results shown below, these surface layers should be oxide layers. According to Figure 13 in Ref. 7, the critical thickness of the oxide layer on the pure element thin-film standards, which causes $5 \%$ of X-ray absorption, is about $1000 \mathrm{~nm}$ for $\mathrm{Fe}$ and $\mathrm{Mn} \mathrm{K}$ lines. Therefore, the influence of the surface oxide layers to determination of the $\zeta$-factors is negligible in terms of absorption. From the above, the thicknesses were $109.37 \mathrm{~nm}$ for Fe thin film and $116.91 \mathrm{~nm}$ for $\mathrm{Mn}$ thin film. The error of thickness $(\Delta t)$ measured by XRR is $0.02 \mathrm{~nm}$. 


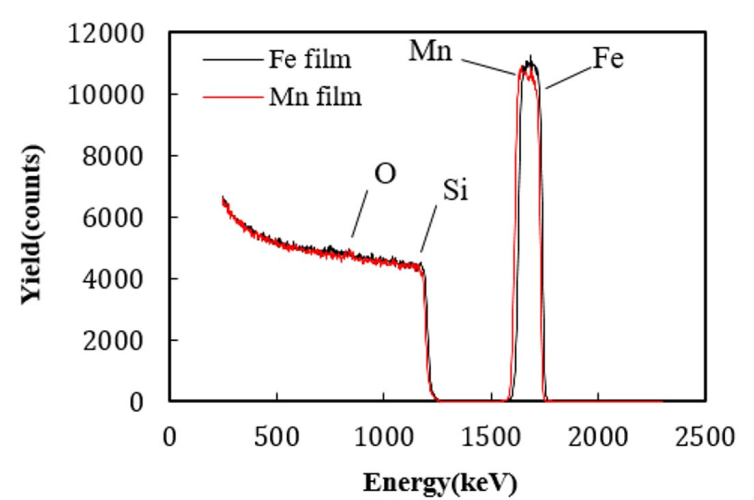

Figure 3: RBS results of $\mathrm{Fe} / \mathrm{Si}$ (black dots) and $\mathrm{Mn} / \mathrm{Si}$ (red dots).

Table 3: Composition (at \%) of the Fe and Mn thin films.

\begin{tabular}{lccc}
\hline & $\mathrm{Fe}$ & $\mathrm{Mn}$ & $\mathrm{O}$ \\
\hline Fe film & $93.5 \pm 3.0$ & - & $6.5 \pm 5.5$ \\
Mn film & - & $96.2 \pm 3.0$ & $(3.8)$ \\
\hline
\end{tabular}

Figure 3 shows the RBS spectra of the $\mathrm{Fe} / \mathrm{Si}$ and $\mathrm{Mn} / \mathrm{Si}$ samples. The peaks for only three elements ( $\mathrm{Fe}$ or $\mathrm{Mn}, \mathrm{Si}$ and O) were observed in the spectra. The yield corresponds to the content of each element. From these data, the composition was calculated as shown in Table 3. Oxygen content in Mn was under detection limit (4.0 at $\%)$. Thus, 3.8 at $\%$ was estimated by the difference of composition of Mn between the measured and theoretical density. Figure 4 shows depth profiles of $\mathrm{Fe}$ or $\mathrm{Mn}, \mathrm{O}$, and $\mathrm{Si}$ estimated by RBS. The depth was estimated by assuming that the film has the theoretical density. The error of composition $\Delta C$ measured by RBS is 3 at $\%$.

SEM images were taken to measure the thickness from the sideview of the samples. Figure 5 shows the cross-sectional SEM images of the metal thin films. The average thickness measured by SEM was $100 \mathrm{~nm}$, while $114 \mathrm{~nm}$ by a surface profiler for Fe. The thickness of Mn film was $121 \mathrm{~nm}$ by SEM and $127 \mathrm{~nm}$ by profiler. In the $\mathrm{Mn}$ film, however, there is considerable surface roughness between the $\mathrm{Mn}$ and Pt boundary consistent with the XRR results.

Since it is difficult to estimate the thickness of bilayer thin films by XRR and SEM, the thickness of the $\mathrm{Mn} / \mathrm{Fe}$ film was estimated by the deposition rate of each film as $100 \mathrm{~nm} / 40$ $\mathrm{nm}$. The thickness of the Mn film which fabricated by "direct deposition method" was $114.5 \mathrm{~nm}$ (measured by the surface profiler). The error of thickness $(\Delta t)$ measured by the surface profiler is $2.1 \mathrm{~nm}$ for the Fe film and $2.4 \mathrm{~nm}$ for the Mn film. The error of $\Delta t$ measured by SEM is estimated to be $10 \mathrm{~nm}$.

\section{B. Determination of the $\zeta$-factor}

The $\zeta$-factors were calculated from Eq. (1). As for the Fe and Mn thin films which are fabricated by "lift-off method", the density $\rho$ measured by XRR, composition $C$ measured by RBS, total electron dose $D$ by Faraday cup, and intensity $I$ by STEM-SDD were used, while the thickness $t$ obtained by
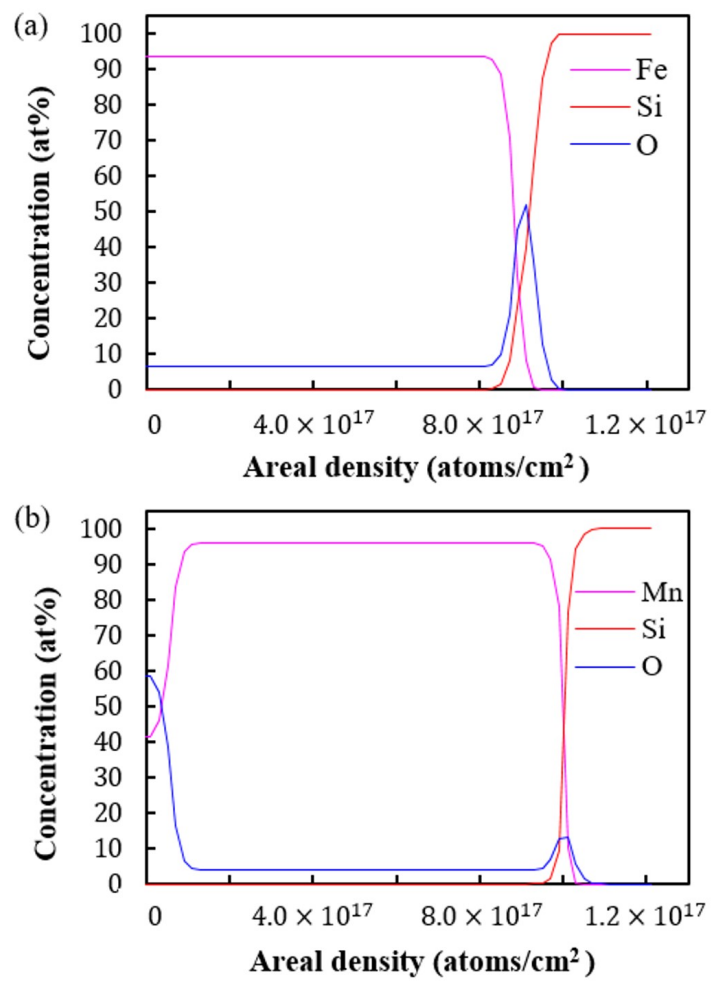

Figure 4: An estimated film depth vs. the elemental profiles of (a) $\mathrm{Fe}, \mathrm{O}$, and $\mathrm{Si}$ in the Fe film, and (b) Mn, O, and Si in the Mn film.

profiler, SEM or XRR were examined. The final values of the $\zeta$-factors for $\mathrm{Fe}$ for our experimental conditions are found to be $\zeta_{\mathrm{Fe}}=227 \pm 4.2 \mathrm{~kg} \mathrm{~m}^{-2}$ (a thickness measured by profiler), $199 \pm 19.4 \mathrm{~kg} \mathrm{~m}^{-2}$ (a thickness by SEM), $218 \pm 0.6 \mathrm{~kg} \mathrm{~m}^{-2}$ (a thickness by XRR). Similarly, the values for the $\zeta$-factors of $\mathrm{Mn}$ are $\zeta_{\mathrm{Mn}}=181 \pm 3.5 \mathrm{~kg} \mathrm{~m}^{-2}$ (a thickness measured by profiler), $172 \pm 14.2 \mathrm{~kg} \mathrm{~m}^{-2}$ (a thickness by SEM), $166 \pm 0.5 \mathrm{~kg}$ $\mathrm{m}^{-2}$ (a thickness by XRR). The $\zeta$-factor estimated from the $\mathrm{Mn} / \mathrm{Fe}$ fabricated by "lift-off method" was calculated and found to be $\zeta_{\mathrm{Mn}}=195 \pm 4.7 \mathrm{~kg} \mathrm{~m}^{-2}$ (a thickness estimated by the deposition rate of the $\mathrm{Mn}$ thin films).

On the other hand, the $\zeta$-factors of the $\mathrm{Fe}$ and the $\mathrm{Mn}$ thin films prepared by "direct deposition method" were $\zeta_{\mathrm{Fe}}=236$ $\pm 5.0 \mathrm{~kg} \mathrm{~m}^{-2}$ and $\zeta_{\mathrm{Mn}}=202 \pm 4.2 \mathrm{~kg} \mathrm{~m}^{-2}$ (a thickness measured by the profiler), respectively.

(a)

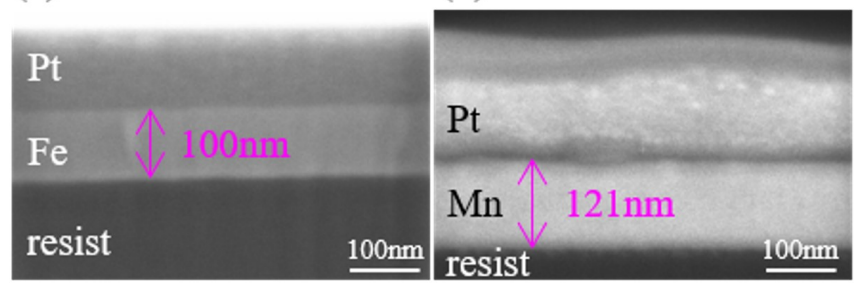

Figure 5: SEM images of a side of Pt/Fe/resist/Si (a), and Pt/Mn/resist/Si (b). The green numbers in both SEM images indicate the film thicknesses 
Table 4: The results of the composition calculated by the $\zeta$-factor method and the Cliff-Lorimer method.

\begin{tabular}{|c|c|c|c|}
\hline & $\mathrm{Fe}(\mathrm{wt} \%)$ & $\operatorname{Mn}(w t \%)$ & $\mathrm{P}(\mathrm{wt} \%)$ \\
\hline Fluorescent X-ray analysis & 98.537 & 1.450 & 0.013 \\
\hline Cliff-Lorimer ratio method & 98.70 & 1.30 & - \\
\hline$\zeta$-factor method (type 1) & $98.92 \pm 0.029$ & $1.08 \pm 0.029$ & - \\
\hline$\zeta$-factor method (type 2 ) & $98.84 \pm 0.035$ & $1.16 \pm 0.035$ & - \\
\hline$\zeta$-factor method (type 3 ) & $98.80 \pm 0.034$ & $1.20 \pm 0.034$ & - \\
\hline
\end{tabular}

It should be noted that the values of the $\zeta$-factors of $\mathrm{Fe}$ showed good coincidence with each other, while the $\zeta$-factors of Mn were varied depending on the fabrication and measurement methods. We calculated the error of the $\zeta$-factor using Eq. (18) in Ref. 7:

$$
\Delta \zeta_{j}^{\mathrm{cal}}=\sqrt{\sum_{i}\left[\zeta_{i}^{\mathrm{cal}}\left(\Delta q_{i}\right)-\zeta_{j}^{\mathrm{cal}}\right]^{2}}
$$

Here, $q_{i}$ represents errors of the intensity, the thickness, the density, and the composition.

\section{Application of the $\zeta$-factors to determine the composition of an iron alloy}

The Fe-Mn-P alloy was prepared as a thin-film specimen having a thickness of about $100 \mathrm{~nm}$ by FIB-SEM. Table 4 shows the results of EDS measurements. The calculated composition by the $\zeta$-factor method [applying Eqs. (2) and (3)] and the C-L ratio method [applying Eqs. (6) and (7)] are listed. In Table 4 , three types of combination of the $\zeta$-factors were compared. In all estimation, the value of $\zeta_{\mathrm{Fe}}$ was fixed to 227 $\pm 4.2 \mathrm{~kg} \mathrm{~m}^{-2}$ (a thickness measured by a profiler), while the values of $\zeta_{\mathrm{Mn}}$ are varied. Table 5 shows the detail of these types; the sample, the film formation, and the measurement or estimation methods for the thickness of the Mn films.

$$
\begin{aligned}
& \frac{C_{\mathrm{A}}}{C_{\mathrm{B}}}=k_{\mathrm{AB}} \frac{I_{\mathrm{A}}}{I_{\mathrm{B}}} \\
& C_{\mathrm{A}}+C_{\mathrm{B}}=1
\end{aligned}
$$

According to the results shown in Table 4, the Mn compositions obtained by the $\zeta$-factor method were smaller than that of $\mathrm{C}$ - $\mathrm{L}$ ratio method in all cases. We calculated the error of compositions using Eq. (19) in Ref. 7:

$\Delta C_{j}=\sqrt{\sum_{k=1}^{n}\left[C_{j}\left(\Delta \zeta_{k}\right)-C_{j}\right]^{2}+\sum_{k=1}^{n}\left[C_{j}\left(\Delta I_{k}\right)-C_{j}\right]^{2}}$

In particular, the composition of Mn determined by the $\zeta$ factor method (type 1) showed relatively large difference from the fluorescent X-ray analysis. It implies that the actual $\zeta$-factor of Mn should be bigger than the calculated value, and that the thickness of the Mn thin film estimated by the present methods was smaller than real value. The $\zeta$-factor of Fe showed good coincidence with two different thin-film approaches except for the case of measurements with SEM
Table 5: The combination of the $\zeta$-factors in Table 4.

\begin{tabular}{lccc}
\hline Type & Sample & Film formation & Thickness \\
\hline 1 & $\mathrm{Mm}$ & Lift-off & Profiler \\
2 & $\mathrm{Mn} / \mathrm{Fe}$ & Lift-off & Rate of film forming \\
3 & $\mathrm{Mn}$ & Direct & Profiler \\
\hline
\end{tabular}

having relativity bigger error than the other methods. The $\zeta$ factor of $\mathrm{Mn}$, on the other hand, were varied although the same measurement methods were utilized for determination of the thickness. If the $\zeta$-factor of Fe was correct, the thickness of Mn thin film should be 135\% lager than the thickness obtained from the surface profiler. It was speculated that bending or overlapping of the Mn film makes such a big difference of thickness.

To overcome the drawback of the Mn single film, we used the $\mathrm{Mn} / \mathrm{Fe}$ sample to prevent the bending or overlapping of Mn film [the $\zeta$-factor method (type 2 )]. In this case, $\zeta_{\text {Mn }}$ estimated by $\mathrm{Mn} / \mathrm{Fe}$ increased as $195 \pm 4.7 \mathrm{~kg} \mathrm{~m}^{-2}$, which is close to the value of $\zeta_{\mathrm{Fe}}$.

Furthermore, in type 3, the "direct deposition method" effectively prevented the overlapping of film. In this case, $\zeta_{\mathrm{Mn}}$ is calculated to be $202 \pm 4.2 \mathrm{~kg} \mathrm{~m}^{-2}$ which is surely close to the values for type 2 . The composition of $\mathrm{Mn}$ in $\mathrm{Fe}-\mathrm{Mn}$ alloy is estimated by the type 3 combination was $1.20 \mathrm{wt} \%$ (Table 4). This value is within $10 \%$ difference from that for the C-L ratio method. This means that this improved $\zeta_{\mathrm{Mn}}$ is good enough for quantitative X-ray analysis. The composition of Mn was a little bit smaller than the that of chemical composition. This might be due to desorption or damage of Mn during the FIB during the sample preparation.

\section{CONCLUSIONS}

In this paper, we proposed two thin-film approaches, namely, the "lift-off method" and the "direct deposition method" to determine the $\zeta$-factors for Fe and Mn. In the proposed methods, the sample thickness can be measured relatively easily compared to the methods in the previous reports. It was suggested that determination of the accurate film thicknesses was essential for the accurate measurement of the $\zeta$ factor. It was also proven that the layered structure and the "direct deposited" thin film are more suitable for standard sample, which can prevent bending and overlapping of the samples. The composition of $\mathrm{Mn}$ in Fe-Mn alloy is estimated to be $1.20 \mathrm{wt} \%$ with $\zeta_{\mathrm{Fe}}=227 \pm 4.2 \mathrm{~kg} \mathrm{~m}^{-2}$ and $\zeta_{\mathrm{Mn}}=202 \pm$ 


\section{ALC '19 Conference}

$4.2 \mathrm{~kg} \mathrm{~m}^{-2}$ (type 3 ). This value was $0.1 \mathrm{wt} . \%$ difference from that of the $\mathrm{C}-\mathrm{L}$ method analysis. This proved that the proposed method to fabricate the standard sample can measure $\zeta$-factors in a good accuracy. Since the method is simple and easily applied to determine $\zeta$-factors for other elements, it gives more feasibility in quantitative $\mathrm{X}$-ray analysis to examine localized light element distribution which needs consideration of X-ray absorption, for example, segregation of phosphorus in grain boundaries of steels.

\section{Acknowledgments}

The authors thank Prof. M. Watanabe of Lehigh University for their advices on the $\zeta$-factor method. This study was carried out as a part of Materials Open Platform (MOP) -Steel project at National Institute for Materials Science (NIMS). The authors sincerely thank Ms. Y. Hara and Ms. A. Nakamura of NIMS for their technical assistance.

Note

This paper was presented at the 12th International Symposium on Atomic Level Characterizations for New Materials and Devices '19 (ALC '19), in conjunction with the 22nd International Conference on Secondary Ion Mass Spectrometry (SIMS-22), Miyako Messe, Kyoto, Japan, 20-25 October, 2019.

\section{References}

[1] P. Lejček, Anal. Chim. Acta 297, 165 (1994).

[2] H. Kimura, Tetsu-to-Hagane 79, 754 (1993) (in Japanese).
[3] M. Yamaguchi, J. Japan Inst. Met. Mater. 72, 657 (2008) (in Japanese).

[4] P. Lejček, S. Hofmann, and V. Paidar, Acta Materi. 51, 3951 (2003).

[5] W. Y.-Qing and C. J. McMahon, Mater. Sci. Technol. 3, 207 (1987).

[6] E. P. Gorzkowski, M. Watanabe, A. M. Scotch, H. M. Chan, and M. P. Harmer, J. Mater. Sci. 39, 6735 (2004).

[7] M. Watanabe and D. B. Williams, J. Microsc. 221, 89 (2006).

[8] D. Zanaga, T. Altantzis, J. Sanctorum, B. Freitag, and S. Bals, Ultramicroscopy 164, 11 (2016).

[9] G. Kothleitner, W. Grogger, M. Dienstleder, and F. Hofer, Microsc. Microanal. 20, 678 (2014).

[10] A. Kitahara, S.Yasuno, and K. Fujikawa, Trans. Mater. Res. Soc. Japan 34, 613 (2009).

[11] D. B. Williams and C. B. Carter, Transmission Electron Microscopy, 2nd ed. (Springer, Boston, 2009) p. 642.

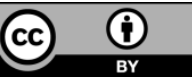

All articles published on e-J. Surf. Sci. Nanotechnol. are licensed under the Creative Commons Attribution 4.0 International (CC BY 4.0). You are free to copy and redistribute articles in any medium or format and also free to remix, transform, and build upon articles for any purpose (including a commercial use) as long as you give appropriate credit to the original source and provide a link to the Creative Commons (CC) license. If you modify the material, you must indicate changes in a proper way.

Published by The Japan Society of Vacuum and Surface Science 